\title{
Quantitative Nano-imaging of Cells with a High Energy X-ray Cryo Nano-probe
}

Yang Yang ${ }^{1, *}$, Sylvain Bohic ${ }^{1,2}$, Florin Fus ${ }^{1}$, Alexandra Pacureanu ${ }^{1}$, Julio Cesar da Silva ${ }^{1}$, Murielle Salome $^{1}$ and Peter Cloetens ${ }^{1}$

${ }^{1 .}$ ESRF - The European Synchrotron, 38000 Grenoble, France

2. Inserm, U1216, University of Grenoble Alpes, Grenoble, France

* Corresponding author, yang.yang@esrf.fr

Metals, present in trace concentrations such as iron, copper and zinc, play a fundamental role in all forms of life. They are essential as enzyme cofactors or cell metabolism regulators. An accurate localization and quantification of these elements in terms of molarity or mass fraction, in two-dimension (2D) and three-dimension (3D), is needed to enrich our understanding of their potential roles in relevant physiological mechanisms, as well as in pathological processes. This requires the appropriate analytical tools with sufficient chemical sensitivity and high spatial resolution at the intra-cellular level.

We will present the recent advances of the ESRF ID16A-NI hard X-ray nanoprobe, optimized for quantitative 3D characterization of the morphology and the elemental composition of specimens in their native state. Located $185 \mathrm{~m}$ from the source, the beamline offers extreme focusing down to $13 \mathrm{~nm}$ with a very high photon flux $\left(10^{12}\right.$ photons/s at $\left.\Delta \mathrm{E} / \mathrm{E} \sim 1 \%\right)$ [1]. Switchable between two discrete energies at $17 \mathrm{keV}$ or $33.6 \mathrm{keV}$, the hard X-ray nano-probe is generated by two respective pairs of fixed-curvature multilayer coated Kirkpatrick-Baez mirrors [2] enclosed in a high vacuum system $\left(\sim 10^{-8} \mathrm{mbar}\right)$.

Three techniques are routinely employed for nano-imaging applications, consisting of scanning X-ray fluorescence microscopy (XRF), phase contrast holo-nanotomography and ptychography. Synchrotron $\mathrm{X}$-ray fluorescence microscopy provides label-free multi-elemental mapping with a spatial resolution down to $30 \mathrm{~nm}$. Complementary to X-ray fluorescence, the two coherent X-ray imaging techniques provide access to the structure by mapping changes in electron density. These approaches can be registered within the same region of interest of the sample to yield quantitative $2 \mathrm{D}$ and even $3 \mathrm{D}$ molarity and mass fraction measurements when extended with tomography techniques. Furthermore, the cellular structures and elemental distributions can be preserved spatially and quantitatively by vitrification and performing the measurements under cryogenic conditions at $120 \mathrm{~K}$.

Recent applications of the single cell quantitative nano-imaging will be presented. With high resolution $\mathrm{XRF}$, we were able to locate and quantify metallodrugs within small organelles in cancer cells down to nanomolar Os concentrations [3]. In another scientific case, XRF was combined with soft X-ray tomography under cryogenic conditions to study the biochemistry of malaria parasite infected red blood cells. The elemental concentrations of $\mathrm{Fe}, \mathrm{S}$ and $\mathrm{K}$, etc. was quantified in various cell compartments with a good precision [4]. Finally, we will show that the XRF measurements in 2D and 3D in conjunction with phase contrast holo-nanotomography can be used to achieve absolute quantification on molarity and mass fraction of elements such as Fe, P and $\mathrm{K}$ in human macrophages [5] and red blood cells.

References:

[1] J. C. Da Silva, A. Pacureanu, Y. Yang, S. Bohic, et al. Optica 4 (2017), p. 492-495. 
[2] C. Morawe, R. Barrett, P. Cloetens, B. Lantelme, et al. Proc. SPIE 9588 (2015) p. 958803.

[3] C. Sanchez- Cano, I. Romero- Canelón, Y. Yang, I. J. Hands- Portman, et al. Chemistry 23 (2017), p. 2512-2516.

[4] S. Kapishnikov, L. Leiserowitz, Y. Yang, P. Cloetens, et al. Scientific reports 7 (2017), p. 802.

[5] C. Gramaccioni, Y. Yang, A. Procopio, A. Pacureanu, et al. Applied Physics Letters 112 (2018) p. 053701.

Figure 1. Schematic layout of the beamline experimental setup. A single undulator line $(\Delta \mathrm{E} / \mathrm{E} \sim 1 \%)$ is focused by a pair of multilayer coated KB mirrors. Samples are scanned through the nano-focus for XRF measurements. Then samples are moved downstream of the focus to record multiple magnified holographic images to retrieve the phase maps.

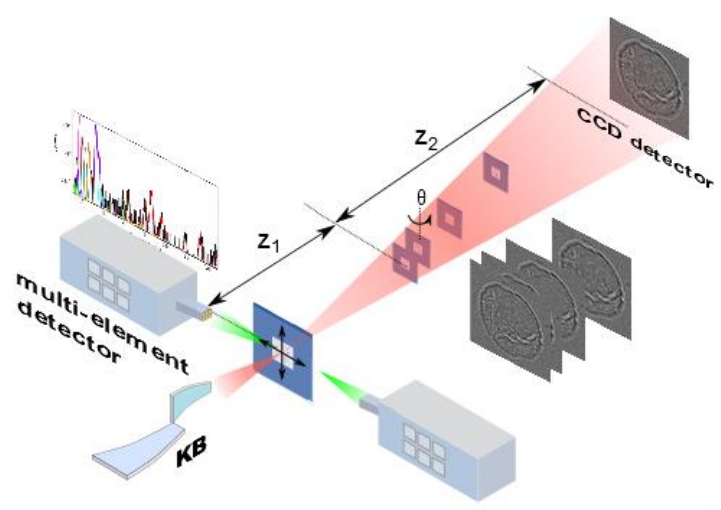

Figure 2. XRF maps of a $500 \mathrm{~nm}$ thick section of ovarian cancer cells (A2780) treated for $24 \mathrm{~h}$ with 1 $\mu \mathrm{M}$ Os drugs, showing the cellular distribution of Os and Ca. Raster scan: $20 \times 20 \mathrm{~nm}^{2}$ step size, $50 \mathrm{~ms}$ dwell time. White ellipses show the absence of $\mathrm{Ca}$ in areas with high density of Os. Scale bar $2 \mu \mathrm{m}$. Calibration bar in $\mathrm{ng} \mathrm{m}^{-2}[3]$.

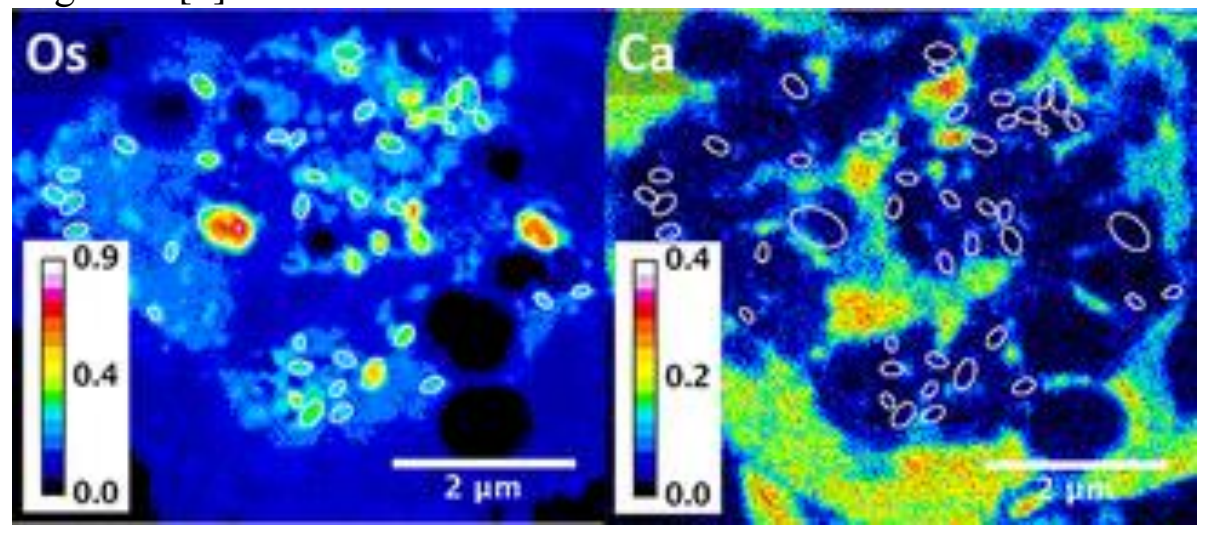

